\title{
Springer Finance
}

Editorial Board

M. Avellaneda

G. Barone-Adesi

M. Broadie

M.H.A. Davis

E. Derman

C. Klüppelberg

E. Kopp

W. Schachermayer 


\section{Springer Finance}

Springer Finance is a programme of books aimed at students, academics and practitioners working on increasingly technical approaches to the analysis of financial markets. It aims to cover a variety of topics, not only mathematical finance but foreign exchanges, term structure, risk management, portfolio theory, equity derivatives, and financial economics.

Ammann M., Credit Risk Valuation: Methods, Models, and Application (2001)

Back K., A Course in Derivative Securities: Introduction to Theory and Computation (2005)

Barucci E., Financial Markets Theory. Equilibrium, Efficiency and Information (2003) Bielecki T.R. and Rutkowski M., Credit Risk: Modeling, Valuation and Hedging (2002)

Bingham N.H. and Kiesel R., Risk-Neutral Valuation: Pricing and Hedging of Financial Derivatives (1998, 2nd ed. 2004)

Brigo D. and Mercurio F., Interest Rate Models: Theory and Practice (2001, 2nd ed. 2006)

Buff R., Uncertain Volatility Models-Theory and Application (2002)

Carmona R.A. and Tehranchi M.R., Interest Rate Models: an Infinite Dimensional Stochastic Analysis Perspective (2006)

Dana R.A. and Jeanblanc M., Financial Markets in Continuous Time (2002)

Deboeck G. and Kohonen T. (Editors), Visual Explorations in Finance with

Self-Organizing Maps (1998)

Delbaen F. and Schachermayer W., The Mathematics of Arbitrage (2005)

Elliott R.J. and Kopp P.E., Mathematics of Financial Markets (1999, 2nd ed. 2005)

Fengler M.R., Semiparametric Modeling of Implied Volatility (2005)

Geman H., Madan D., Pliska S.R. and Vorst T. (Editors), Mathematical

Finance-Bachelier Congress 2000 (2001)

Gundlach M., Lehrbass F. (Editors), CreditRisk ${ }^{+}$in the Banking Industry (2004)

Kellerhals B.P., Asset Pricing (2004)

Külpmann M., Irrational Exuberance Reconsidered (2004)

Kwok Y.-K., Mathematical Models of Financial Derivatives (1998)

Malliavin P. and Thalmaier A., Stochastic Calculus of Variations in Mathematical

Finance (2005)

Meucci A., Risk and Asset Allocation (2005)

Pelsser A., Efficient Methods for Valuing Interest Rate Derivatives (2000)

Prigent J.-L., Weak Convergence of Financial Markets (2003)

Schmid B., Credit Risk Pricing Models (2004)

Shreve S.E., Stochastic Calculus for Finance I (2004)

Shreve S.E., Stochastic Calculus for Finance II (2004)

Yor M., Exponential Functionals of Brownian Motion and Related Processes (2001)

Zagst R., Interest-Rate Management (2002)

Zhu Y.-L., Wu X., Chern I.-L., Derivative Securities and Difference Methods (2004)

Ziegler A., Incomplete Information and Heterogeneous Beliefs in Continuous-time Finance (2003)

Ziegler A., A Game Theory Analysis of Options (2004) 
Eric Jondeau, Ser-Huang Poon and Michael Rockinger

Financial Modeling Under Non-Gaussian Distributions

望 Springer 


\author{
Eric Jondeau \\ University of Lausanne and Swiss \\ Finance Institute \\ HEC-Department of Finance and \\ Insurance \\ CH-1015 Lausanne-Dorigny
}

Switzerland

\section{Michael Rockinger \\ University of Lausanne and Swiss \\ Finance Institute \\ HEC-Department of Finance and \\ Insurance}

CH-1015 Lausanne-Dorigny

Switzerland
Ser-Huang Poon

Manchester Business School

University of Manchester

Oxford Road

Manchester M13 9PL

UK

Mathematics Subject Classification (2000): 91B28, 91B84

British Library Cataloguing in Publication Data

A catalogue record for this book is available from the British Library

Library of Congress Control Number: 2006923899

Springer Finance Series ISSN 1616-0533

ISBN-10 1-84628-419-8

Printed on acid-free paper

ISBN-13 978-1-84628-419-9

(C) 2007 Springer-Verlag London Limited

MATLAB ${ }^{\circledR}$ is a trademark of The MathWorks, Inc. and is used with permission. The MathWorks does not warrant the accuracy of the text or exercises in this book. This book's use or discussion of MATLAB ${ }^{\circledR}$ software or related products does not constitute endorsement or sponsorship by The MathWorks of a particular pedagogical approach or particular use of the MATLAB ${ }^{\circledR}$ software.

Apart from any fair dealing for the purposes of research or private study, or criticism or review, as permitted under the Copyright, Designs and Patents Act 1988, this publication may only be reproduced, stored or transmitted, in any form or by any means, with the prior permission in writing of the publishers, or in the case of reprographic reproduction in accordance with the terms of licences issued by the Copyright Licensing Agency. Enquiries concerning reproduction outside those terms should be sent to the publishers.

The use of registered names, trademarks, etc. in this publication does not imply, even in the absence of a specific statement, that such names are exempt from the relevant laws and regulations and therefore free for general use.

The publisher makes no representation, express or implied, with regard to the accuracy of the information contained in this book and cannot accept any legal responsibility or liability for any errors or omissions that may be made.

Printed in the United States of America

(MVY)

\section{$\begin{array}{lllllllll}9 & 8 & 7 & 6 & 5 & 4 & 3 & 2 & 1\end{array}$}

Springer Science+Business Media, LLC springer.com 
To my family from Eric Jondeau

In loving memory of Janet and Harry
from Ser-Huang Poon

To Laura

from Michael Rockinger 


\section{Preface}

Practitioners and academic researchers who have handled financial market data know that asset return distributions do not have the bell shape that is associated with the Gaussian or normal distribution. The fact that many popular models are still based on the assumption of normality is because of the simplicity that the Gaussian model presents, and perhaps the bigger reason is the lack of understanding of the modeling, estimation, and handling of nonGaussian distributions. The use of Gaussian models, when the asset return distributions are not normal, can be very dangerous; such a practice could lead to a wrong choice of portfolio, the underestimation of extreme losses, and hugely mispriced derivative products. Non-Gaussian distributions are the key theme of this book, which addresses the causes and the consequences of non-normality of asset returns.

Other books in this field include Campbell, Lo, and Mackinlay (1997), and Embrechts, Klüppelberg, and Mikosch (1997). In related fields, one may also cite Gouriéroux and Jasiak (2001), Tsay (2002), and Taylor (2005). On option pricing, we have the book of Schoutens (2003), and of Cont and Tankov (2004). All these books are complementary to this one and are useful for understanding some aspects of the modeling of asset returns or option prices. In this book, we cover a wide range of issues that are resulting from nonnormality and time dependency of both asset returns and option prices.

The book is written for non-mathematicians who want to model financial market prices. Its emphasis is on practice. We worked hard to make the materials accessible to non-mathematicians but at the same time not to sacrifice the mathematical rigor and the complexity of the original models. This book targets practitioners in the finance industry and especially those who call themselves Quant and have the responsibility of managing portfolios and monitoring financial risk. We also hope the not-so-Quant people will find this book useful in understanding the myth of rocket scientists' involvement in quantitative finance. This book is suitable for use as a core text for specialist M.Sc. and Ph.D. students in Empirical Finance, Financial Econometrics and 
Financial Derivatives. It is useful for mathematicians who want to know more about how their mathematical tools are applied in finance and for those who just want to know more about finance and financial markets. We provide, in Part V, some mathematical appendices that we feel are sufficient for coping with the mathematical contents of the main parts. Some basic knowledge in statistics, calculus, and probability plus plenty of determination should see the readers through the core materials. Most of the material presented in this book is extracted from our papers and material used to teach practitioners and generations of M.Sc. and Ph.D. students at University of Lausanne and Manchester Business School.

One of our main aims is to bridge the gap between the theoretical developments and the practical implementations of what many users and researchers perceived as "sophisticated" models or black boxes. We offer many empirical illustrations of the models described in this book, and especially those developed in Parts II and III. ${ }^{1}$ Although we use only a small collection of stock market data in these examples due to the space constraint, many of the techniques and models described here could equally be applied to other financial time series, such as exchange and interest rates. But it is important to remember that models are meant to capture stylized facts. Different types of financial assets may have slightly or very different characteristics. Another disclaimer is of importance: Although we argue and provide evidence that there is a need to incorporate non-normality and time dependency in the modeling of asset returns, we do not suggest that this should always be the case. Moreover, we would not be held responsible for any problem the reader may encounter in the implementation of these techniques.

Finally, all the figures and estimations reported in this book have been produced using MATLAB. ${ }^{2}$ This software provides a very efficient optimization routine, and we have made available on our web sites many MATLAB codes that are used in this book.

Eric Jondeau and Michael Rockinger both want to thank the Ecole des HEC of the University of Lausanne, the International Center for Financial Asset Management and Engineering (FAME), and the Swiss Finance Institute (SFI) for their constant support. They thank many generations of M.Sc. and Ph.D. students for the many discussions, comments and questions. Ser-Huang Poon wants to thank the Manchester Business School, and the Manchester Accounting and Finance Group in particular, for providing much support.

${ }^{1}$ Daily data used in this book comes from Datastream, and intradaily data comes from the Euronext Paris dataset.

${ }^{2}$ MATLABC is a registered trademark of The MathWorks, Inc. For MATLAB product information, please contact: The MathWorks, Inc. 3 Apple Hill Drive, Natick, MA 01760-2098, USA. E-mail: info@mathworks.com. Web: www.mathworks.com. 
She wants to thank also her students for many stimulating discussions and grilling reading classes. All three authors express their gratitude to Laura Rockinger for reading the entire manuscript and correcting many mistakes. We express our thanks to many other people, colleagues, friends, and family, to whom we are most grateful for their support. They are too many to name here.

Eric Jondeau, Ser-Huang Poon, and Michael Rockinger August 2006 


\section{Contents}

\section{Part I Financial Markets and Financial Time Series}

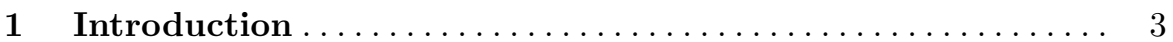

1.1 Financial markets and financial time series............. 3

1.2 Econometric modeling of asset returns $\ldots \ldots \ldots \ldots \ldots \ldots .4$

1.3 Applications of non-Gaussian econometrics ............ 5

1.4 Option pricing with non-Gaussian distributions $\ldots \ldots \ldots \ldots . \quad 5$

2 Statistical Properties of Financial Market Data $\ldots \ldots \ldots \ldots$

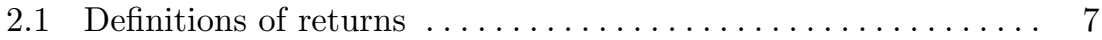

2.1.1 Simple returns ......................... 8

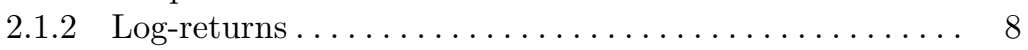

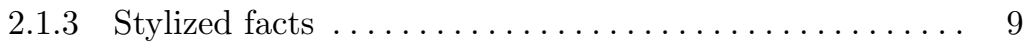

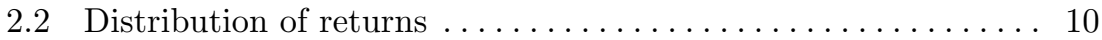

2.2.1 Moments of a random variable $\ldots \ldots \ldots \ldots \ldots \ldots \ldots$

2.2 .2 Empirical moments . . . . . . . . . . . . . . . . . 14

2.2 .3 Testing for normality $\ldots \ldots \ldots \ldots \ldots \ldots \ldots \ldots \ldots \ldots$

2.3 Time dependency ........................... 21

2.3.1 Serial correlation in returns . . . . . . . . . . . . 22

2.3.2 Serial correlation in volatility $\ldots \ldots \ldots \ldots \ldots \ldots \ldots .23$

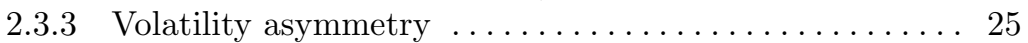

2.3.4 Time-varying higher moments............... 26

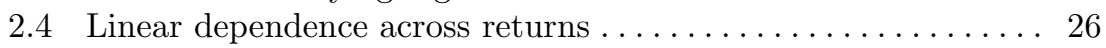

2.4.1 Pearson's correlation coefficient ................ 27

2.4.2 Test for equality of two correlation coefficients ...... 28

2.4.3 Test for equality of two correlation matrices ....... 30

2.5 Multivariate higher moments................... 31

2.5.1 Multivariate co-skewness and co-kurtosis . ........ 31

2.5.2 Computing moments of portfolio returns ......... 32 
3 Functioning of Financial Markets and Theoretical Models

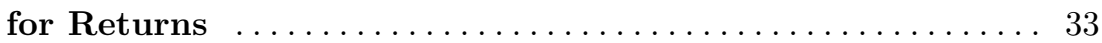

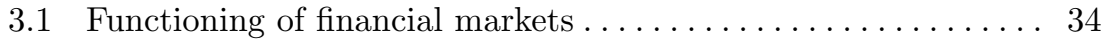

3.1 .1 Organization of financial markets ............. 34

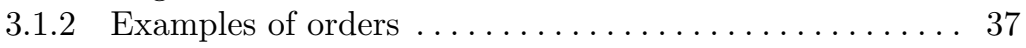

3.1.3 Components of the bid-ask spread ........... 39

3.2 Mandelbrot and the stable distribution . . . . . . . . . . . . 39

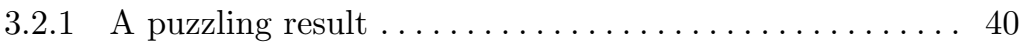

3.2 .2 Stable distribution $\ldots \ldots \ldots \ldots \ldots \ldots \ldots \ldots \ldots \ldots \ldots 4$

3.3 Clark's subordination model .................... 44

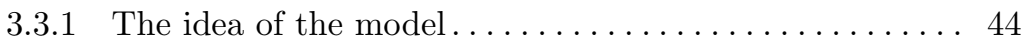

3.3.2 The density of returns under subordination . . . . . . . 46

3.4 A bivariate mixture-of-distribution model for return and

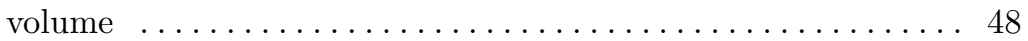

3.4.1 A microstructure model for information arrivals . . . . . 48

3.4.2 Implications of the mixture of distributions hypothesis . 53

3.4.3 Testing the mixture of distribution hypothesis...... . 57

3.4.4 Extensions ...................... 61

3.5 A model of prices and quotes in a quote-driven market . . . . . 62

3.5.1 A model based on the trade flow . . . . . . . . . . 63

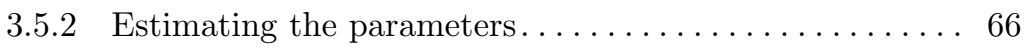

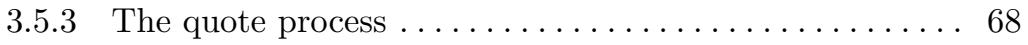

3.5.4 Extension to the liquidation of a large portfolio . . . . . 73

\section{Part II Econometric Modeling of Asset Returns}

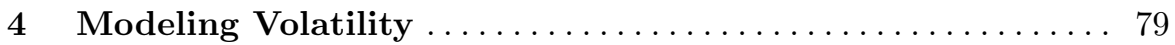

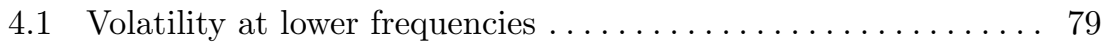

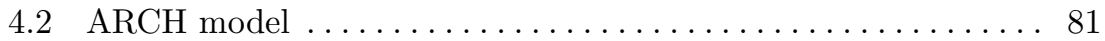

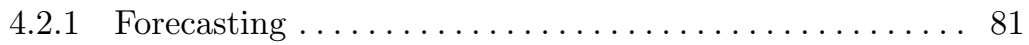

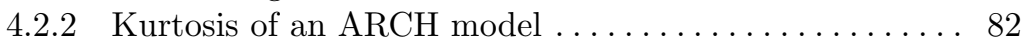

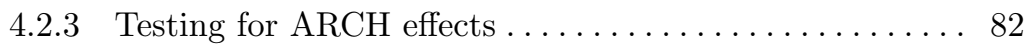

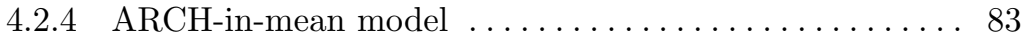

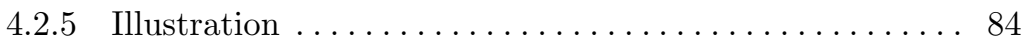

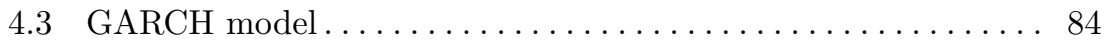

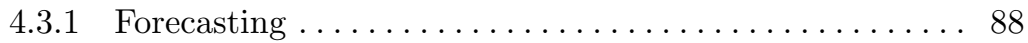

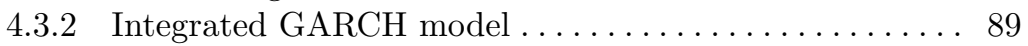

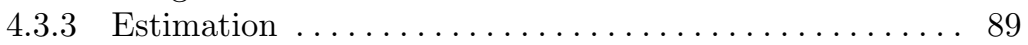

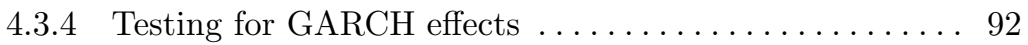

4.3.5 Software to estimate ARCH and GARCH models ..... 92

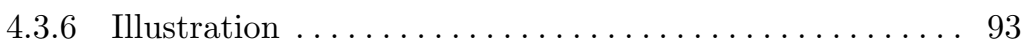

4.4 Asymmetric GARCH models . . . . . . . . . . . . . . . . 94

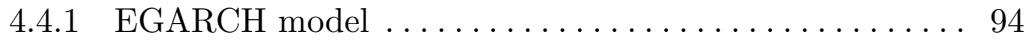

4.4 .2 TGARCH model . . . . . . . . . . . . . . . . 95 
4.4 .3 GJR model . . . . . . . . . . . . . . . . . . . . . . . . 95

4.4 .4 Cox-Box transform ....................... 95

4.4.5 News impact curve ................... 96

4.4.6 Partially non-parametric estimation.............. 96

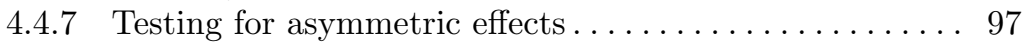

4.4 .8 Illustration $\ldots \ldots \ldots \ldots \ldots \ldots \ldots \ldots \ldots \ldots \ldots \ldots \ldots$

4.5 GARCH model with jumps . . . . . . . . . . . . . . . . . 99

4.5.1 A model with time-varying jump intensity . . . . . . . . . 101

4.5.2 An empirical illustration ..................... 105

4.6 Aggregation of GARCH processes . . . . . . . . . . . . . 108

4.6.1 Temporal aggregation ........................ 109

4.6.2 Cross-sectional aggregation . . . . . . . . . . . . . . . . 113

4.6.3 Estimation of the weak GARCH process............ 114

4.7 Stochastic volatility ........................... 115

4.7.1 From GARCH models to stochastic volatility models . . . 115

4.7.2 Estimation of the discrete time SV model ......... 117

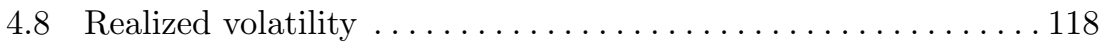

4.8.1 The difficulty to disentangle jumps . . . . . . . . . . 119

4.8.2 Quadratic variation.......................... 123

4.8.3 Power variation ............................. 124

4.8.4 Bipower variation . ...................... 126

4.8.5 Estimation over finite time intervals $\ldots \ldots \ldots \ldots \ldots 128$

4.8.6 Realized covariance ....................... 135

4.8.7 Further related results . . . . . . . . . . . . . . . . 141

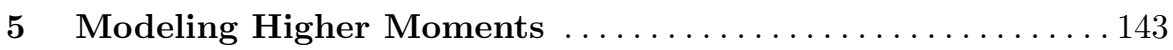

5.1 The general problem ............................ 144

5.1 .1 Higher moments of a GARCH process.............. 145

5.1.2 Quasi Maximum Likelihood Estimation ............. 148

5.1.3 The existence of distribution with given moments .... 151

5.2 Distributions with higher moments $\ldots \ldots \ldots \ldots \ldots \ldots \ldots \ldots \ldots \ldots \ldots$

5.2 .1 Semi-parametric approach . ................. 153

5.2.2 Series expansion about the normal distribution ....... 155

5.2 .3 Skewed Student $t$ distribution ................. 159

5.2.4 Generating asymmetric distributions ............. 166

5.2 .5 Pearson IV distribution $\ldots \ldots \ldots \ldots \ldots \ldots \ldots \ldots \ldots$

5.2 .6 Entropy distribution ......................... 172

$5.3 \quad$ Specification tests and inference .................... 177

5.3.1 Moment specification tests................... 177

5.3.2 Adequacy tests based on density forecasts . . . . . . . . 179

5.3.3 Adequacy tests based on interval forecasts ......... 180

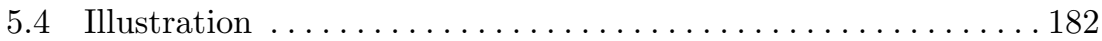

5.5 Modeling conditional higher moments .............. 188

5.5.1 Tests for autoregressive conditional higher moments . . 189

5.5.2 Modeling higher moments directly ................ 189 
5.5.3 Modeling the parameters of the distribution

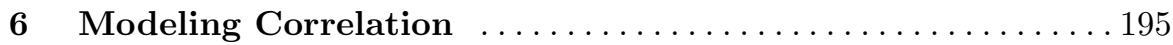

6.1 Multivariate GARCH models . . . . . . . . . . . . . . . . . . 197

6.1.1 Vectorial and diagonal GARCH models . . . . . . . . 198

6.1.2 Dealing with large-dimensional systems . . . . . . . . 200

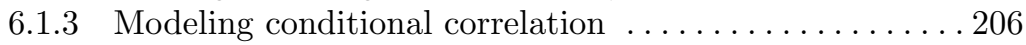

6.1 .4 Estimation issues . . . . . . . . . . . . . . . 210

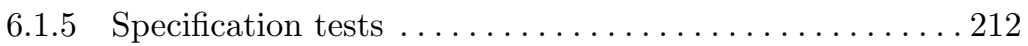

6.1.6 Test of constant conditional correlation matrix . . . . . . 214

6.1 .7 Illustration . . . . . . . . . . . . . . . . . . . . . . . . 217

6.2 Modeling the multivariate distribution . . . . . . . . . . . 223

6.2 .1 Standard multivariate distributions . . . . . . . . . . 225

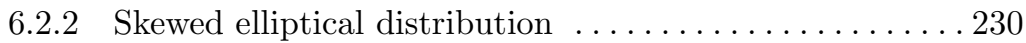

6.2 .3 Skewed Student $t$ distribution . . . . . . . . . . . . 233

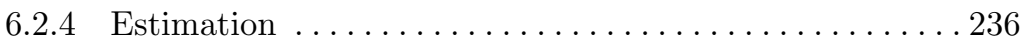

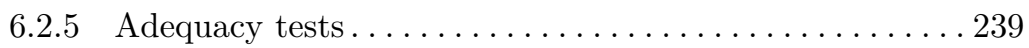

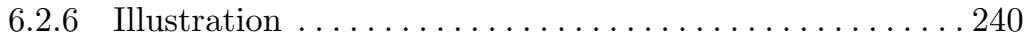

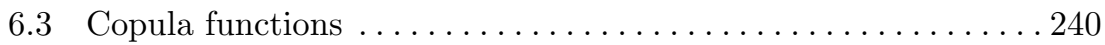

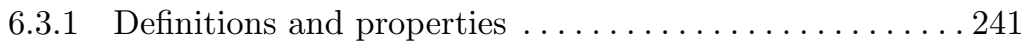

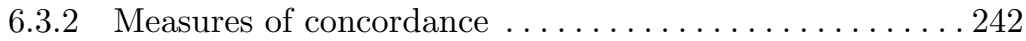

6.3 .3 Non-parametric copulas . . . . . . . . . . . . . . . . . 244

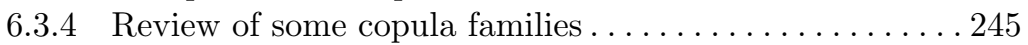

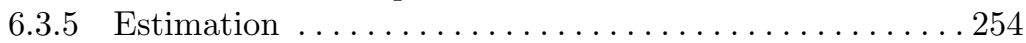

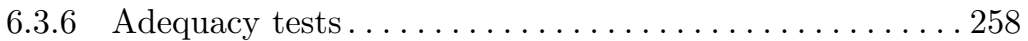

6.3.7 Modeling the conditional dependency parameter. . . . . . 259

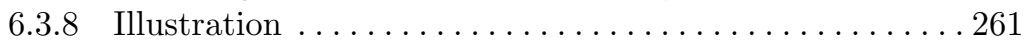

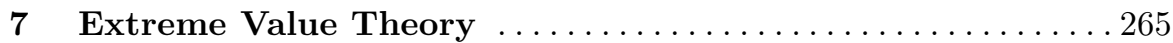

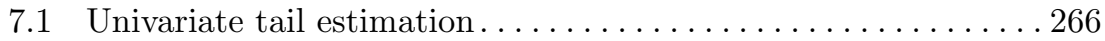

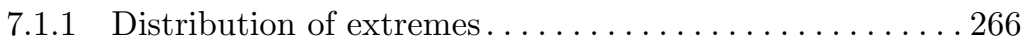

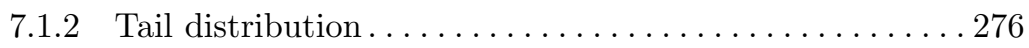

7.1 .3 The case of weakly dependent data . . . . . . . . . . 291

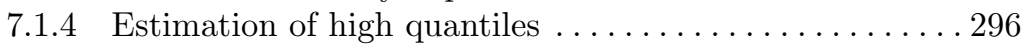

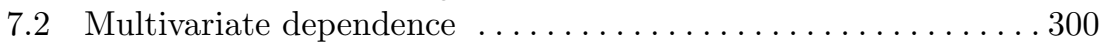

7.2.1 Characterizing tail dependency ................ 303

7.2.2 Estimation and statistical inference on $\bar{\chi}$ and $\chi \ldots \ldots 307$

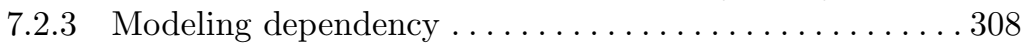

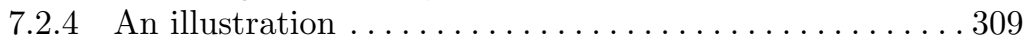

7.2 .5 Further investigations .................... 311 


\section{Part III Applications of Non-Gaussian Econometrics}

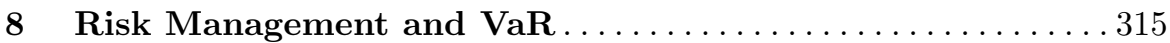

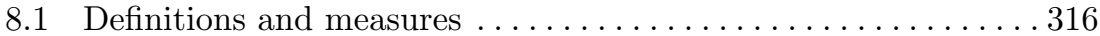

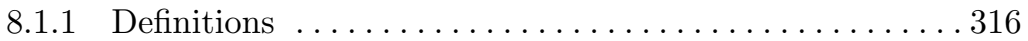

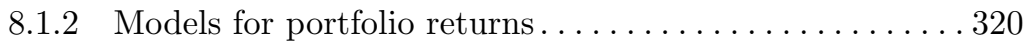

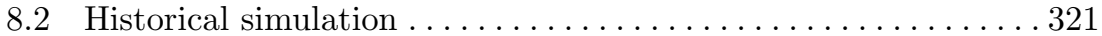

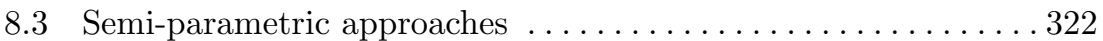

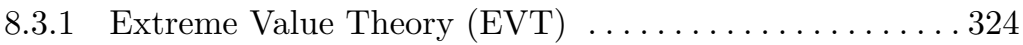

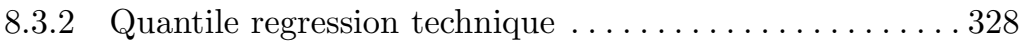

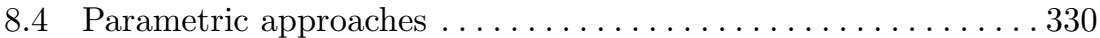

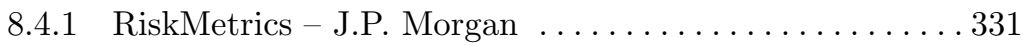

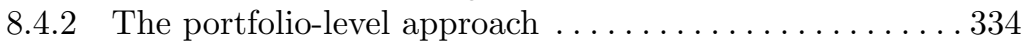

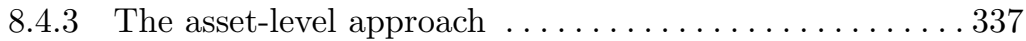

8.5 Non-linear models . . . . . . . . . . . . . . . . . . . . . . 341

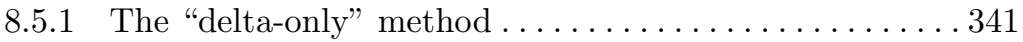

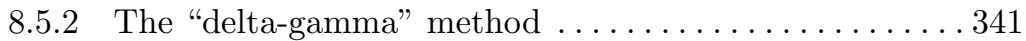

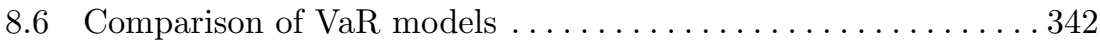

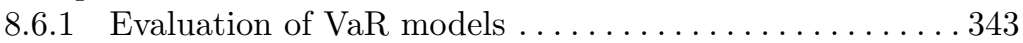

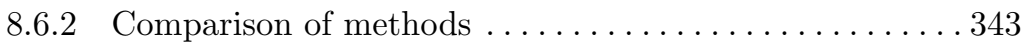

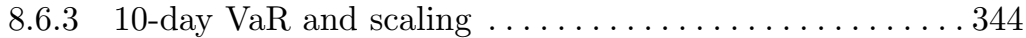

8.6 .4 Illustration . . . . . . . . . . . . . . . . . . 345

9 Portfolio Allocation . . . . . . . . . . . . . . . . . . . . 349

9.1 Portfolio allocation under non-normality . . . . . . . . . . . . . 349

9.1.1 Direct maximization of expected utility . . . . . . . 350

9.1.2 An approximate solution based on moments . . . . . . 353

9.2 Portfolio allocation under downside risk . . . . . . . . . . . 359

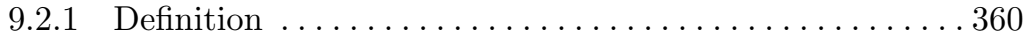

9.2 .2 Downside risk as an additional constraint . . . . . . . . 360

9.2.3 Downside risk as an optimization criterion . . . . . . . . 361

\section{Part IV Option Pricing with Non-Gaussian Returns}

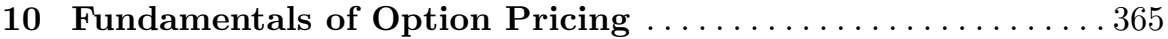

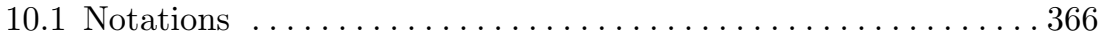

10.2 The no-arbitrage approach to option pricing . . . . . . . . 369

10.2.1 Choice of a stock price process . . . . . . . . . . . . . 369

10.2.2 The fundamental partial differential equation . . . . . . 371

10.2.3 Solving the fundamental PDE . . . . . . . . . . . . 373

10.2.4 The Black-Scholes-Merton formula.... . . . . . . . . 375

10.3 Martingale measure and BSM formula . . . . . . . . . . . . 377

10.3.1 Self-financing strategies and portfolio construction . . . . 377 


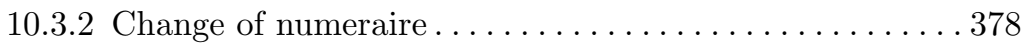

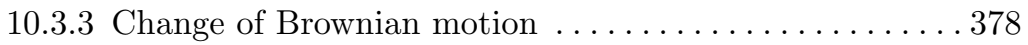

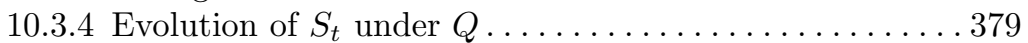

10.3.5 The expected pay-off as a martingale . . . . . . . . 379

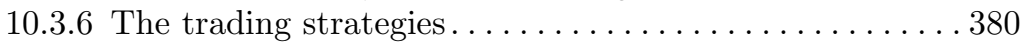

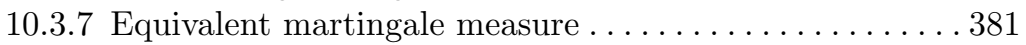

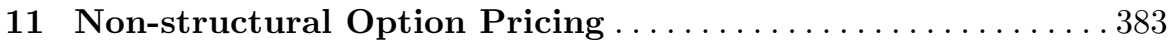

11.1 Difficulties with the standard BSM model . . . . . . . . . . . 384

11.2 Direct estimation of the risk-neutral density . . . . . . . . . 385

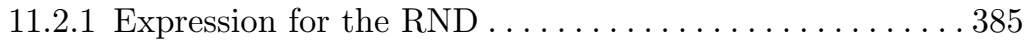

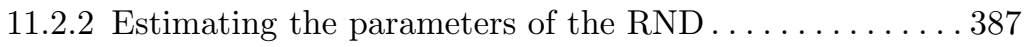

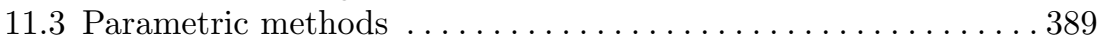

11.3.1 Mixture of log-normal distributions . . . . . . . . . . . 389

11.3.2 Mixtures of hypergeometric functions . . . . . . . . . . 394

11.3.3 Generalized beta distribution . . . . . . . . . . . . . 395

11.4 Semi-parametric methods . . . . . . . . . . . . . . . . . . . 395

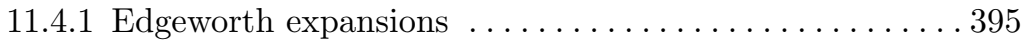

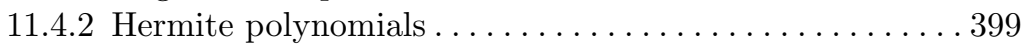

11.5 Non-parametric methods . . . . . . . . . . . . . . . . . . . 402

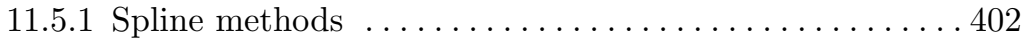

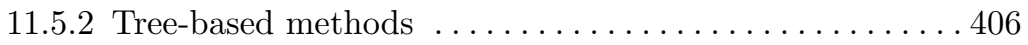

11.5.3 Maximum entropy principle............... 407

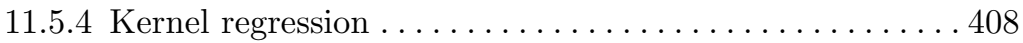

11.6 Comparison of various methods . . . . . . . . . . . . . . 409

11.7 Relationship with real probability . . . . . . . . . . . . 414

11.7.1 The link between RNDs and objective densities . . . . . 414

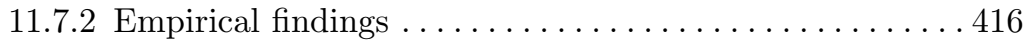

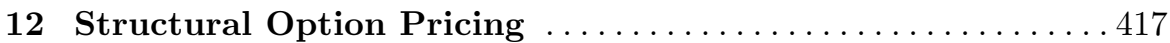

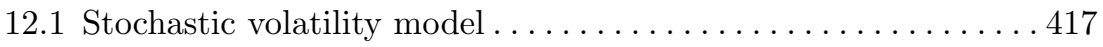

12.1.1 The square root process $\ldots \ldots \ldots \ldots \ldots \ldots \ldots \ldots \ldots \ldots$

12.1.2 Solving the PDE based on characteristic function . . . . . 419

12.1.3 A new partial differential equation ............ . . . . . . . . 22

12.2 Option pricing with stochastic volatility . . . . . . . . . 425

12.2.1 Hull and White $(1987,1988) \ldots \ldots \ldots \ldots \ldots \ldots \ldots \ldots 425$

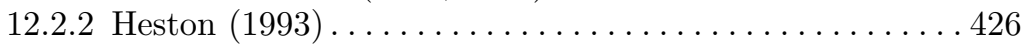

12.2.3 Characteristic function of the SV model . . . . . . . . . 428

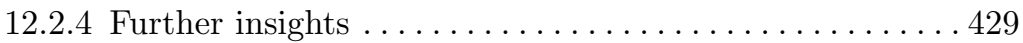

12.3 Models with jumps . . . . . . . . . . . . . . . . . 432

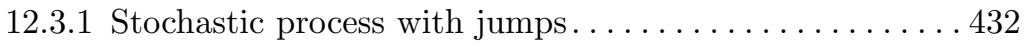

12.3.2 Diffusion with double exponential jumps ......... . 434

12.3.3 Combining stochastic volatility with jumps . . . . . . 436

12.3.4 Jumpy affine models . . . . . . . . . . . . . . . . . . . . 440

12.4 Models with even wilder jumps: Lévy option pricing . . . . . . . 441 
12.4.1 Commonly used Lévy processes . . . . . . . . . . . . 443

12.4.2 Choice of the time-changing process . . . . . . . . . 444

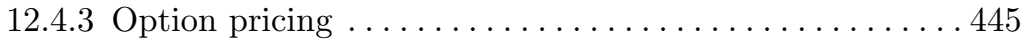

12.4.4 Pricing options with risk-neutral characteristic function 446

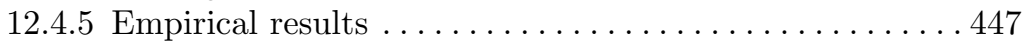

\section{Part V Appendices on Option Pricing Mathematics}

13 Brownian Motion and Stochastic Calculus . . . . . . . . . . 451

13.1 Law of large numbers and the central limit theorem . . . . . . . 451

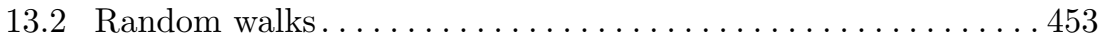

13.3 Construction of the Brownian motion .............. 453

13.4 Properties of the Brownian motion . . . . . . . . . . . . 456

13.5 Stochastic integration ... . . . . . . . . . . . . . . 457

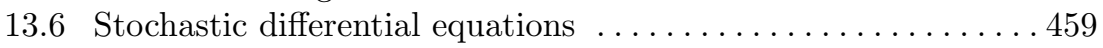

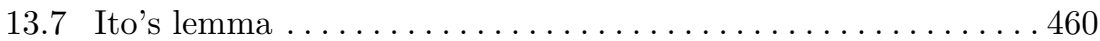

13.8 Multivariate extension of Ito's lemma . . . . . . . . . . . 462

13.9 Transition probabilities and partial differential equations . . . . 463

13.10 Kolmogorov backward and forward equations . . . . . . . . . 464

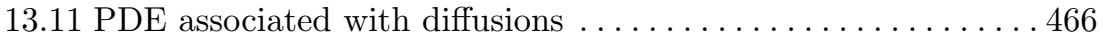

13.12 Feynman-Kac formula . . . . . . . . . . . . . . 468

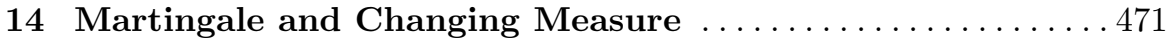

14.1 Martingales . . . . . . . . . . . . . . . . . . . . 471

14.2 Changing probability of a normal distribution . . . . . . . . . 472

14.3 Radon-Nikodym derivative. . . . . . . . . . . . . . . . 473

14.4 Girsanov's theorem . . . . . . . . . . . . . . . . . . . . . . . . . . . . . 474

14.5 Martingale representation theorem . . . . . . . . . . . . 475

15 Characteristic Functions and Fourier Transforms . . . . . . 477

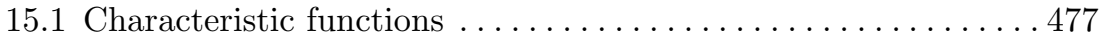

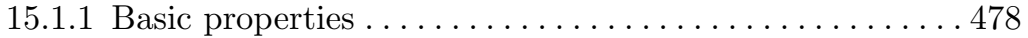

15.1.2 Moments and the characteristic function .........448

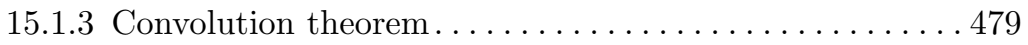

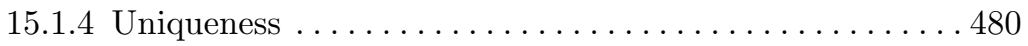

15.1.5 Inversion theorem . . . . . . . . . . . . . . . . . . 480

15.2 Fourier transform and characteristic function............ 483

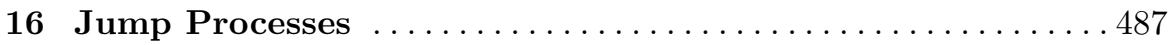

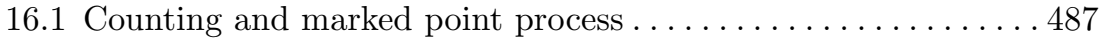

16.2 The Poisson process . . . . . . . . . . . . . . . . 489

16.2.1 Construction of the Poisson distribution . . . . . . . . 489

16.2.2 Properties of the Poisson distribution . . . . . . . . . 491

16.2.3 Moments of pure Poisson process . . . . . . . . . . 492 
XVIII Contents

16.2.4 Compound Poisson process ................ 493

16.3 The exponential distribution . . . . . . . . . . . . . . . 494

16.3.1 Definition and properties ................. 494

16.3.2 Moments of the exponential variable . . . . . . . . 495

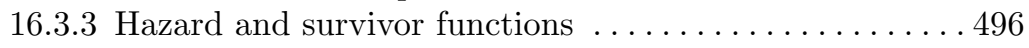

16.4 Duration between Poisson jumps . . . . . . . . . . . . . . . 497

16.5 Compensated Poisson processes . . . . . . . . . . . . . . 498

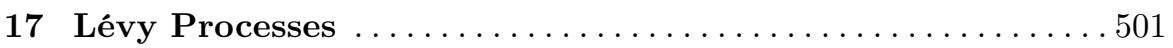

17.1 Construction of the Lévy process . . . . . . . . . . . . 501

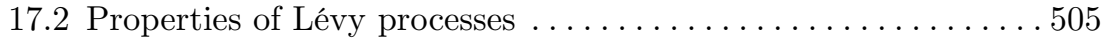

References.............................. 507

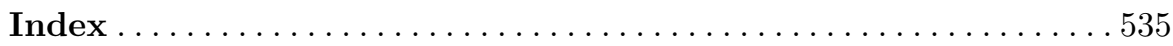

